\title{
On some applications of a class of totally positive bases
}

\author{
Laura Gori and Francesca Pitolli \\ Dip. Me.Mo.Mat. - Università di Roma "La Sapienza" \\ v. A. Scarpa 10, I-00161 Rome
}

\section{Introduction}

It is well known that total positivity plays an important role in many fields, from the design of curve to approximation theory [3, 9, 17]. In recent years there have been many papers on the shape preserving properties of totally positive (TP) bases, in particular for the spaces of polynomials, or for the spaces of polynomial spline functions. We recall that a system of functions is called totally positive if its collocation matrices are TP, i.e., all its minors are non-negative. On the other hand, refinable functions are essential in the construction both of stationary subdivision schemes (and thus in CAGD) $[5,8]$ and of wavelet bases, whose applications to several approximation problems has proved to be very profitable. In particular, recently, problems involving partial differential or integral equations was successfully dealt with by means of wavelets, as a number of paper shows $[1,6,7,16,19]$.

Thus, it is apparent the importance which, in many contexts, the use of totally positive systems of refinable functions may assume. The introduction of a large class of these systems is due to Goodman and Micchelli [11], who, among other things, proved that the system of functions constituted by the integer translates of a refinable function, associated with a left-half plane stable symbol, is totally positive. In the following we shall denote by LHP the set of such refinable functions.

The aim of this paper is to present some applications of a class of totally positive systems of refinable function belonging to LHP, which was introduced in $[13,15]$ and which are explicitly identified by their regularity and symmetry.

The paper is organized as follows; in Section 2, the basic properties of the just quoted refinable functions are reviewed; Section 3 concerns construction and properties of $B$-basis [2, 4] on a given finite interval, starting from a finite set of integer translates of the same refinable function. In Section 4, we introduce two positive approximation operators in terms of the obtained 
B-basis and discuss a few of their properties. In particular, we are interested in analyzing how the use of these functions influences the behavior of certain linear operators of Schoenberg-Bernstein type, which turn out to be shape preserving when they are based on suitable bases of polynomials or spline functions [10].

\section{Preliminaries}

The most typical example of refinable functions belonging to LHP is provided by the cardinal B-spline $M_{n}$, of degree $n$, convolution of $n+1$ times the characteristic function $\chi$ of the interval $[0,1]$

$$
M_{n}=\chi * \chi * \ldots * \chi
$$

it satisfies the general refinement equation

$$
\varphi(x)=\sum_{k \in \mathbb{Z}} a_{k} \varphi(2 x-k),
$$

when the mask $\left\{a_{k}\right\}_{k \in \mathbb{Z}}$ is given by

$$
\begin{cases}a_{k}=\frac{1}{2^{n}}\left(\begin{array}{c}
n+1 \\
k
\end{array}\right), & k=0,1, \ldots, n+1, \\
a_{k}=0, & \text { otherwise. }\end{cases}
$$

Its symbol (or discrete Fourier transform), given by

$$
b(z)=\frac{1}{2^{n}}(1+z)^{n+1}
$$

with $z=e^{i \theta}$, is clearly left-hand plane stable. Moreover, $M_{n}$ belongs to $C^{n-1}(\mathbb{R})$, is supported on $[0, n+1]$ and is symmetric with respect to $(n+1) / 2$.

In [13] another set of refinable functions, which share many properties with the cardinal B-splines, have been introduced. Their masks are explicitly identified by the conditions on $\varphi$ to be centrally symmetric and to belong to LHP and $C^{n-2}(\mathbb{R})$.

It is a one-parameter family of refinable functions satisfying (2.2) for

$$
\begin{cases}a_{k}=\frac{1}{2^{h}}\left[\left(\begin{array}{c}
n+1 \\
k
\end{array}\right)+4\left(2^{h-n}-1\right)\left(\begin{array}{l}
n-1 \\
k-1
\end{array}\right)\right], & k=0,1, \ldots, n+1, \\
a_{k}=0, & \text { otherwise }\end{cases}
$$

whose symbol is given by

$$
p_{n}(z)=\frac{1}{2^{h}}(1+z)^{n-1}\left(z^{2}+\left(2^{h-n+2}-2\right) z+1\right) .
$$


Here, $h \geq n \geq 2$ is a real parameter.

These refinable functions, to be denoted by $\varphi_{n, 1, h}$, can be considered as a generalization of $M_{n}$; in fact, their masks, which are a linear combination of the masks of the cardinal B-spline of degree $n$ and $n-2$, reduce to the mask of $M_{n}$ when $h=n$. For any $n$, and $h \geq n$, the functions $\varphi_{n, 1, h}$ belong to $C^{n-2}(\mathbb{R})$, are compactly supported on $[0, n+1]$ and central symmetric; moreover, the functions $\left\{\varphi_{n, 1, h}(x-j)\right\}_{j \in \mathbb{Z}}$ are linearly independent and provide a totally positive system on $\mathbb{R}$.

In Fig. 1 the graph of $\varphi_{3,1, h}$ for different values of $h$ are displayed, confirming that, when $h$ diverges, $\varphi_{n, 1, h}$ tends to $M_{n-2}$ (cf. (2.5)).

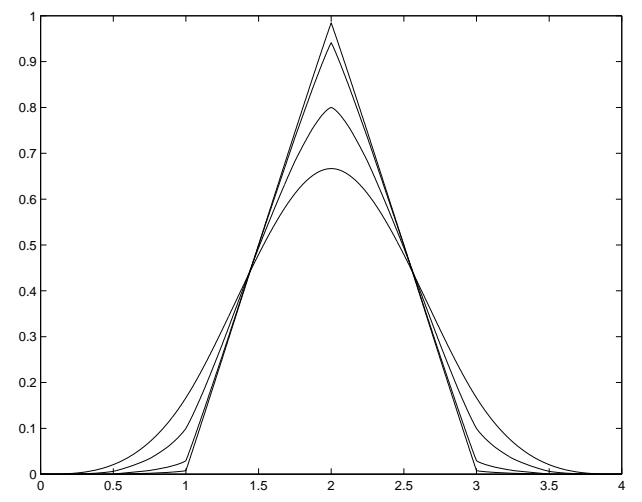

Fig. 1. The refinable functions $\varphi_{3,1, h}$ for $h=3,4,6,8$.

A further generalization was given in [15] where new families of refinable functions $\varphi$ belonging to LHP are identified in terms of their masks, imposing the conditions $\varphi \in C^{n-2 m}(\mathbb{R}), \operatorname{supp} \varphi=[0, n+1], 1 \leq m \leq[n / 2]$, beyond central symmetry. These masks are, again, a linear combination of the masks of the cardinal B-splines of degree $n, n-2, \ldots, n-2 m$, and are expressed by

$$
\begin{cases}a_{k}=\sum_{r=0}^{m} b_{r}^{(r)}\left(\begin{array}{c}
n+1-2 r \\
k-r
\end{array}\right), & k=0, \ldots, n+1, \\
a_{k}=0, & \text { otherwise }\end{cases}
$$

where the coefficients $b_{l}^{(r)}$ are defined recursively as follows:

$b_{l}^{(r)}=b_{l}^{(r-1)}-\left(\begin{array}{c}2 m-2 r+2 \\ l-r+1\end{array}\right) b_{r-1}^{(r-1)}, \quad r=0,1, \ldots, m-1, \quad l=r+1, \ldots, m$,

and $b_{l}^{(0)}, l=0,1, \ldots, 2 m$, are positive numbers such that

$$
\begin{cases}b_{2 m-r}^{(0)}=b_{r}^{(0)}, & r=0,1, \ldots, 2 m \\ b_{m}^{(0)}=2^{2 m-n}-2 \sum_{j=0}^{m-1} b_{j}^{(0)}, & \\ \operatorname{det}\left(b_{2 j-i}^{(0)} ; i, j=1, \ldots, p\right)>0, & p=1, \ldots, 2 m .\end{cases}
$$


(We assume $\left(\begin{array}{l}l \\ i\end{array}\right)=0$ for $i=0$ or $i>l$.)

Their symbol is given by

$$
p(z)=(z+1)^{n-2 m+1} q_{2 m}(z),
$$

where

$$
q_{2 m}(z)=\sum_{k=0}^{2 m} b_{k}^{(0)} z^{k}, \quad q_{2 m}(1)=2^{-n+2 m} .
$$

We explicitly observe that relations (2.9) depend on the conditions on $\varphi$ to be central symmetric and to have a left-half plane stable symbol.

For any fixed $m$, there are $m$ real parameters, namely, $b_{j}^{(0)}, j=0,1, \ldots$, $m-1$, satisfying (2.9). For computational purpose, it is convenient to express them by means of dyadic fractions. For $m=2$ we assume

$$
b_{0}^{(0)}=2^{-h_{1}}, \quad b_{1}^{(0)}=2^{h_{2}-h_{1}},
$$

where the parameter vector $H=\left[h_{1}, h_{2}\right]$ satisfies the condition $h_{1}>n-2+$ $\log _{2}\left(1+2^{h_{2}-1}\right)$ in order to fulfill (2.9). Correspondingly, (2.7) gives

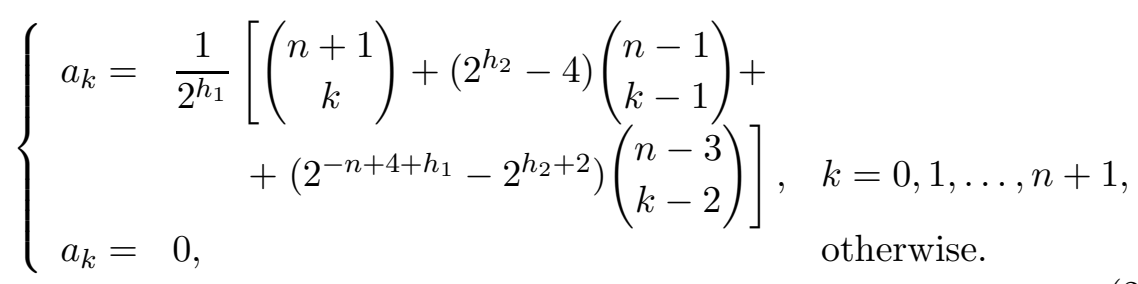

For $h_{1}=n$ and $h_{2}=2$, we get the mask of $M_{n}$.

In the following we shall denote by $H=\left[h_{1}, h_{2}, \ldots, h_{m}\right]$ the vector of the real parameters, by $\varphi_{n, m, H}$ the corresponding refinable functions and by $\Psi$ the set of all functions $\varphi_{n, m, H}$ for any admissible $n, m, H$ :

$$
\Psi=\left\{\varphi_{n, m, H}(x), \operatorname{supp} \varphi=[0, n+1]\right\} .
$$

In Fig. 2 some graphs of $\varphi_{6,2, H}$ for a few values of $n$ and $H$ are displayed.

We conclude listing some properties of the refinable functions $\varphi_{n, m, H}$ and of their masks, which will be useful in the sequel. For the sake of simplicity we suppress the subscripts. There results

$$
\sum_{j \in \mathbb{Z}} \varphi(x-j)=1, \quad x \in \mathbb{R} .
$$

The functions of the system $\{\varphi(x-j), j \in \mathbb{Z}\}$ are linearly independent and there results

$$
\underset{l, j=1, \ldots, r}{\operatorname{det}} \varphi\left(x_{l}-i_{j}\right) \geq 0
$$


for all $x_{1}<x_{2}<\ldots<x_{r}, i_{1}<i_{2}<\ldots<i_{r}, x_{l} \in \mathbb{R}, i_{l} \in \mathbb{Z}$, with the strict inequality holding if and only if $i_{l}<x_{l}<i_{l}+n, l=1, \ldots, r$,

$$
\int_{\mathbb{R}} \varphi(x) d x=1
$$

and

$$
\sum_{k \in \mathbb{Z}} a_{k+2 j}=1, \quad a_{k}=a_{n+1-k} .
$$

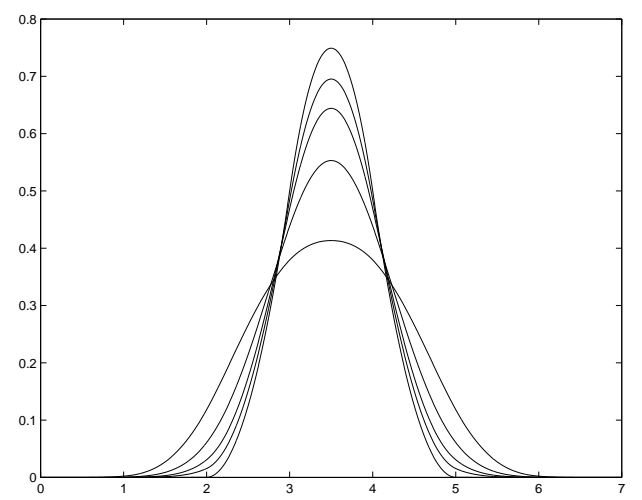

Fig. 2. The refinable function $\varphi_{6,2, H}$ for $h_{1}=6,7,8,9,15$ and $h_{2}=3$.

\section{B-bases of refinable functions}

The properties quoted in Section 2, allow us for concluding that considering any finite interval $I=[\alpha, \beta], \alpha$ and $\beta$ integers, the linearly independent functions of the system

$$
\Phi_{n, m, H}=\left\{\varphi_{n, m, H}(x-j), N_{1} \leq j \leq N_{2}\right\},
$$

where $N_{1}=\alpha-n$ and $N_{2}=\beta-1$, form a normalized (or blending) totally positive (NTP) basis for the space $S$ they span.

As a consequence, the system $\Phi_{n, m, H}$ can be used in order to define a curve which reproduces the shape of a polygonal arc $\Gamma=A_{N_{1}} A_{N_{1}+1} \ldots A_{N_{2}}$ in $\mathbb{R}^{2}$, where $A_{i}=\left(x_{i}, y_{i}\right), i=N_{1}, N_{1}+1, \ldots, N_{2}$, are given points. In fact, consider the curve

$$
\mathbf{r}(t)=\left(r_{1}(t), r_{2}(t)\right)=\sum_{i=N_{1}}^{N_{2}} A_{i} \varphi_{n, m, H}(x-i), \quad x \in I ;
$$

then, the following proposition holds, as a particular case of some results in [10].

Proposition 3.1 The curve $\mathbf{r}$ crosses any straight line $L$ the same number of times the polygonal arc $\Gamma$ crosses $L$; if $\Gamma$ is convex, such is the curve $\mathbf{r}$. 
Thus, the bases $\Phi_{n, m, H}$, for any compatible $n, m, H$, have shape preserving properties, as in the case of polynomial bases and spline function bases discussed in $[3,10]$.

At this point, it is worthwhile to recall that for the space $\mathbb{P}_{n}$ of polynomials of degree less or equal to $n$, the Bernstein basis has optimal shape preserving properties among all NTP bases of $\mathbb{P}_{n}$ on a compact interval, as well as it happens for the B-spline bases in the spaces of polynomial spline functions. Now, in [4] both these bases have been inserted in the more general framework of the $B$-bases, of which they are particular cases. We recall that a TP system of linearly independent functions $u$ defined on $I \subseteq \mathbb{R}$ is said to be a B-basis if all TP bases $v$ of the space generated by $u$ satisfy the relation

$$
v^{T}=u^{T} A,
$$

where $A$ is a nonsingular TP matrix.

In [4], normalized B-bases (or optimal bases) are characterized through certain their properties and an algorithm for their construction is given. This algorithm can be applied, in particular, when the NTP system under consideration is constituted by suitable integer shifts of a refinable function.

Details on the construction of normalized B-bases starting from refinable functions are given in [14]; in Fig. 3 and 4 we show some examples concerning particular choices of $\varphi_{n, m, H}$.

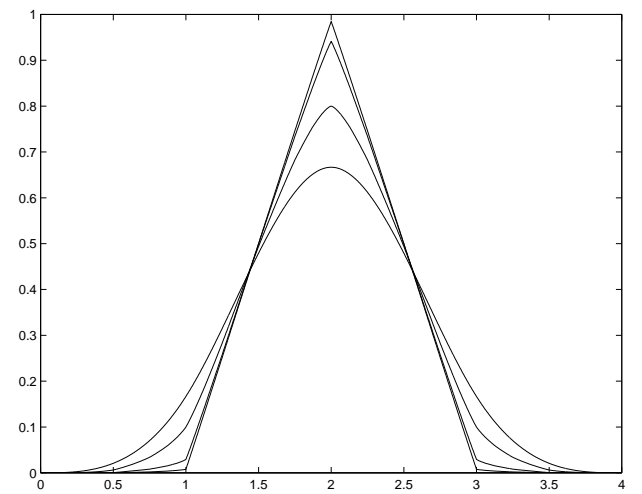

Fig. 3. The NTP basis $\Phi_{3,1,4}$ (dashed line) and the corresponding normalized B-basis (solid line) in the interval $[0,4]$. 


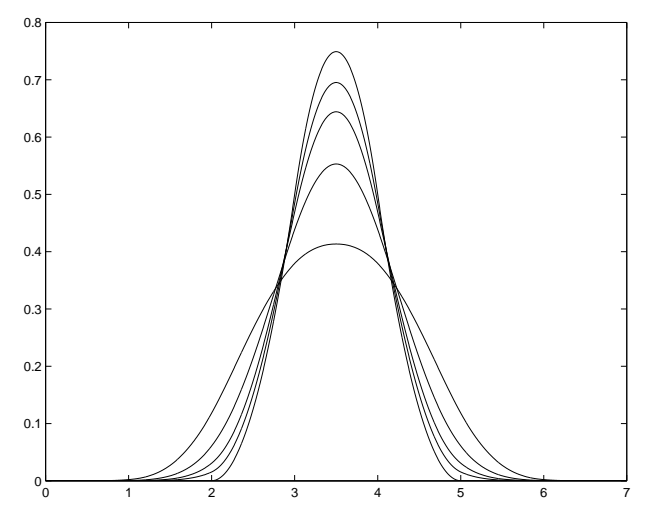

Fig. 4. The NTP basis $\Phi_{5,2,6,2}$ (dashed line) and the corresponding normalized B-basis (solid line) in the interval $[0,6]$.

It is worthwhile to note that when $\varphi_{n, m, H}$ reduces to $M_{n}$, the normalized B-basis obtained from $\left\{M_{n}(x-j)\right\}_{j=N_{1}}^{N_{2}}$ is exactly the cardinal B-spline basis as defined in [21].

\section{Some applications}

Let us consider again the system $\left\{\varphi_{n, m, H}(x-j), j \in \mathbb{Z}\right\}$. The spaces $V_{j}$ spanned by $\left\{2^{j / 2} \varphi_{n, m, H}\left(2^{j} x-k\right)\right\}_{k \in \mathbb{Z}}$ give a multiresolution analysis in $L^{2}(\mathbb{R})$ and $V_{0} \supseteq \mathbb{P}_{n-2 m}[15]$. Based on this property, the following results are interesting for later use.

Proposition 4.1 Let $\varphi$ be any function in $\Psi, n \geq 2 m-1$; then, there results

$$
x=\sum_{j \in \mathbb{Z}}\left(j+\frac{n+1}{2}\right) \varphi(x-j), \quad x \in \mathbb{R} .
$$

Proof. Let us denote by $\mu_{k}$ the $k$-th moment of $\varphi$ :

$$
\mu_{k}=\int_{\mathbb{R}} x^{k} \varphi(x) d x .
$$

From [18, Lemma 5.2] it follows

$$
x=\sum_{j \in \mathbb{Z}}\left(j+\mu_{1}\right) \varphi(x-j), \quad x \in \mathbb{R},
$$

while from $[7$, Sect. 4], one gets

$$
\mu_{1}=\frac{\mu_{0}}{2} \sum_{k=0}^{n+1} k a_{k}
$$


The properties (2.17) and (2.18) give $\mu_{0}=1, \sum_{k=0}^{n+1} a_{k}=2, a_{k}=a_{n+1-k}$; therefore, in the case when $n=2 r$, one can write

$$
\sum_{k=0}^{n+1} k a_{k}=\sum_{k=0}^{r}(2 r+1) a_{k}=2 r+1=n+1,
$$

while for $n=2 r+1$, one has

$$
\begin{aligned}
\sum_{k=0}^{n+1} k a_{k}= & \sum_{k=0}^{r}(2 r+2) a_{k}+(r+1) a_{r+1}= \\
& =(r+1)\left(2 \sum_{k=0}^{r} a_{k}+a_{r+1}\right)=2 r+2=n+1,
\end{aligned}
$$

then (4.1) follows.

The proposition above implies the existence, for any finite NTP system $\Phi_{n, m, H}$ given by (3.1), of $N:=N_{2}-N_{1}+1$ numbers $\xi_{j}, j=N_{1}, \ldots, N_{2}$, such that

$$
x=\sum_{j=N_{1}}^{N_{2}} \xi_{j} \varphi_{n, m, H}(x-j) .
$$

We note that not all $\xi_{j}$ belong to $I$. However, if we consider the B-basis associated with a given $\Phi_{n, m, H}$, then relation (4.2) holds for numbers $\eta_{j} \in I$, $j=N_{1}, N_{1}+1, \ldots, N_{2}$, as the theorem below states.

Theorem 4.2 Let $W_{n, m, H}=\left\{w_{j, n, m, H}\right\}_{j=N_{1}}^{N_{2}}$ be the B-basis generated by the NTP system $\Phi_{n, m, H}$ on the interval I. Then, there exist $N$ numbers $\eta_{N_{1}}, \ldots, \eta_{N_{2}} \in I$ such that

$$
x=\sum_{j=N_{1}}^{N_{2}} \eta_{j} w_{j, n, m, H}(x), \quad x \in I .
$$

Proof. By the notation $X=\left[\xi_{N_{1}}, \ldots, \xi_{N_{2}}\right]^{T},(4.2)$ can be written as

$$
x=\Phi_{n, m, H}^{T} X,
$$

which, by the change of basis $\Phi_{n, m, H}^{T}=W_{n, m, H}^{T} A$ (cf. (3.3)), yields

$$
x=W_{n, m, H}^{T} A X=W_{n, m, H}^{T} Y=\sum_{j=N_{1}}^{N_{2}} \eta_{j} w_{j, n, m, H}(x),
$$

where $Y=A X:=\left[\eta_{N_{1}}, \ldots, \eta_{N_{2}}\right]^{T}$. The sequence $\left\{\xi_{j}\right\}_{j=N_{1}}^{N_{2}}$ is clearly increasing, then such is the sequence $\left\{\eta_{j}\right\}_{j=N_{1}}^{N_{2}}$, seen the properties of $A$; moreover, one has

$$
a=\sum_{j=N_{1}}^{N_{2}} \eta_{j} w_{j, n, m, H}(a)=\eta_{N_{1}}, \quad b=\sum_{j=N_{1}}^{N_{2}} \eta_{j} w_{j, n, m, H}(b)=\eta_{N_{2}},
$$


since, by construction, $w_{j, n, m, H}(a)=\delta_{j, N_{1}}, w_{j, n, m, H}(b)=\delta_{j, N_{2}}$.

Consider now the B-basis $W_{n, m, H}$ of the space $S$ spanned by $\left\{\varphi_{n, m, H}(x-\right.$ $j)\}$ on $I$; for any real valued function $f$ on $I$, the following operator analogous to the Bernstein-Schoenberg operator [20] can be defined:

$$
S_{1} f(x)=\sum_{j=N_{1}}^{N_{2}} f\left(\eta_{j}\right) w_{j, n, m, H}(x) .
$$

$S_{1}$ is clearly a linear positive operator for which the following theorem holds, where $S^{-}(f)$ denotes the number of strict sign changes of $f$ on $I$, that is $S^{-}(f)=\sup S^{-}\left(f\left(x_{0}\right), \ldots, f\left(x_{r}\right)\right)$, where the supremum is taken over all increasing sequences $\left(x_{0}, \ldots, x_{r}\right)$ in $I$, for all $r$.

Theorem 4.3 The operator $S_{1}$ reproduces any linear function, satisfies the end-point interpolation property

$$
S_{1} f(\alpha)=f(\alpha), \quad S_{1} f(\beta)=f(\beta)
$$

for any function $f$ on $I$. Moreover, for any linear function $l$ and any function $f$ on $I$, there results

$$
S^{-}\left(S_{1} f-l\right) \leq S^{-}(f-l)
$$

Proof. Theorem 4.2 assures the existence of a sequence $\left\{\eta_{j}\right\}_{j=N_{1}}^{N_{2}}$, such that

$$
\alpha=\eta_{N_{1}} \leq \eta_{N_{1}+1} \leq \ldots \leq \eta_{N_{2}}=\beta
$$

for which (4.3) holds; then, (4.5) and (4.6) follow from some results in [10].

In Fig. 5 we display the graphs of $S_{1} f$ and $f$ for a particular choice of $\varphi_{n, m, H}$ and $f$. The points $f\left(\eta_{j}\right)$ are represented by the symbol $*$.

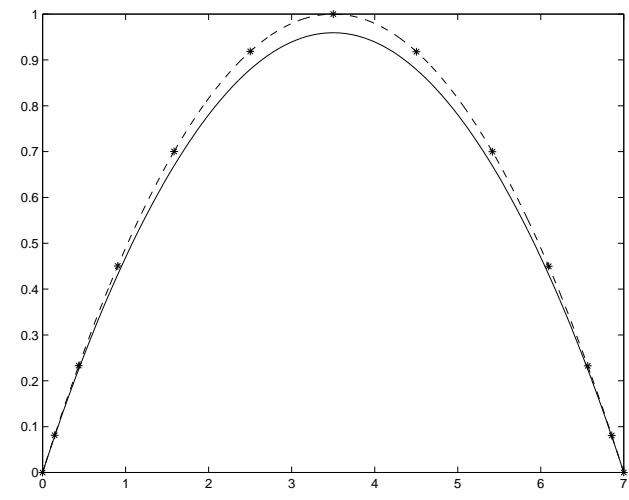

Fig. 5. The operator $S_{1} f(x)$ relative to $\varphi_{6,4,7,3}$ (solid line) and the function $f(x)=1-(2 x / 7-1)^{2}$ in the interval $[0,7]$. 
It has already been remarked that any system $\Phi_{n, m, H}$ on a given interval $I$, can be considered as a generalization of the basis $\left\{M_{n}(x-j)\right\}_{j=N_{1}}^{N_{2}}$, and the B-basis $W_{n, m, H}$ corresponding to $\varphi_{n, m, H}$ as a generalization of the cardinal B-spline basis. Therefore, it is rather natural to consider another linear positive operator in terms of the system $W_{n, m, H}$, namely

$$
S_{2} f(x)=\sum_{j=N_{1}}^{N_{2}}\left(\int_{\alpha}^{\beta} b_{j, n-2} f\right) w_{j, n, m, H}, \quad x \in I,
$$

where $b_{j, n-2}$ is the $j$-th element of the B-basis corresponding to $\Phi_{n-2, m, K}$, $K=\left[h_{1}-2, h_{2}, \ldots, h_{m}\right]$, normalized so to have unit integral. We adopt the convention

$$
\int_{\alpha}^{\beta} b_{N_{1}, n-2} f=f(\alpha), \quad \int_{\alpha}^{\beta} b_{N_{2}, n-2} f=f(\beta) .
$$

In the case when $W_{n, m, H}$ reduces to the B-spline basis, $S_{2} f$ is the positive operator introduced in [12]. In the more general case a number of numerical experiments shows that the following relatin holds

$$
\int_{\alpha}^{\beta} b_{j, n-2}(t) t d t=\eta_{j}
$$

where $\eta_{j}$ are the numbers in (4.3). The (4.3) and (4.9) justify the following conjecture.

Conjecture. The linear positive operator $S_{2}$ reproduces any linear function, satisfies the end-point interpolation conditions

$$
S_{2}(f) f(\alpha)=f(\alpha), \quad S_{2} f(\beta)=f(\beta)
$$

for any $f$ on $I$; moreover $S^{-}\left(S_{2} f-l\right) \leq S^{-}(f-l)$ for any linear function $l$.

In Fig. 6 the graphs of $S_{2} f$ confirms our conjecture for a particular choice of $\varphi_{n, m, H}$.
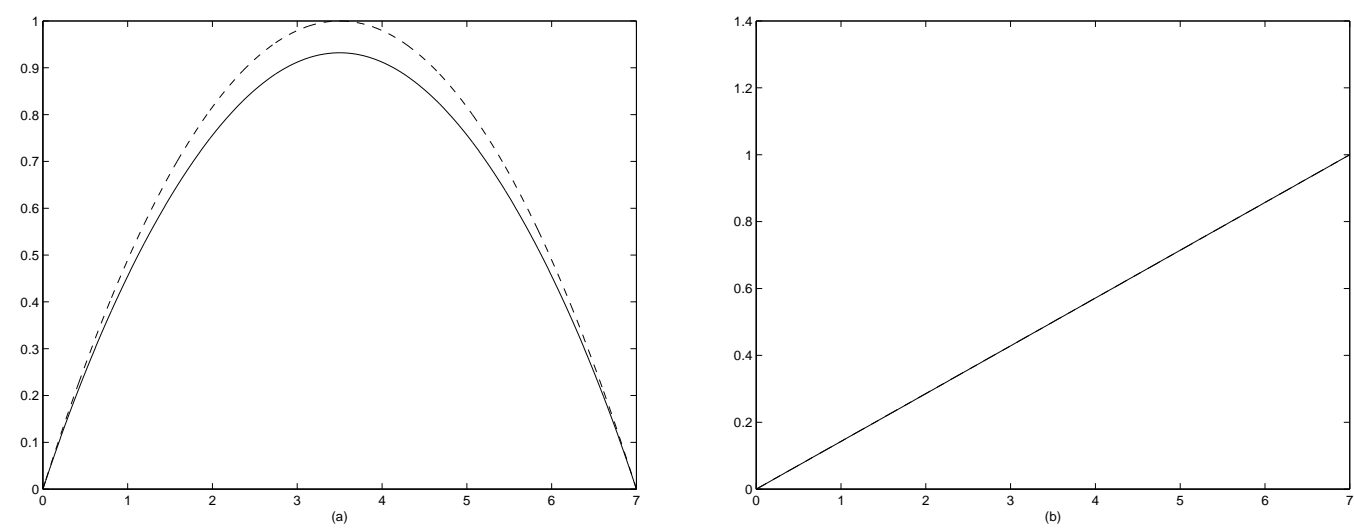

Fig. 6. The operator $S_{2} f(x)$ relative to $\varphi_{6,4,7,3}$ (solid line) for the functions $f(x)=1-(2 x / 7-1)^{2}$ (solid line) (a) and $f(x)=x / 7$ (b) in the interval $[0,7]$. 


\section{References}

[1] Canuto, C., A. Tabacco, and K. Urban, The wavelet element method. Construction and analysis. Appl. Comput. Harmon. Anal. 6 (1999), $1-52$.

[2] Carnicer, J.M., and J.M. Peña, Shape preserving representation and optimality of the Bernstein basis, Adv. Comput. Math. 1 (1993), 173196.

[3] Carnicer, J.M., and J.M. Peña, Totally positive bases for shape preserving curves design and optimality of B-splines, Comput. Aided Geom. Design 11 (1994), 633-654.

[4] Carnicer, J.M., and J.M. Peña, Total positivity and optimal bases, in Total Positivity and its Applications (M. Gasca and C.A. Micchelli eds.), Kluwer Academic Publishers, 1996, 133-155.

[5] Cavaretta, A.S., W. Dahmen and C.A. Micchelli, Stationary subdivision, Mem. Am. Math. Soc. 93 (1991).

[6] Chen, Z., C.A. Micchelli and Y. Xu, The Petrov-Galerkin method for second kind integral equations. II. Multiwavelets schemes, Adv. Comput. Math. 7 (1997), 199-233.

[7] Dahmen, W., and C. A. Micchelli, Using the refinement equation for evaluating integrals of wavelet, SIAM J. Numer. Anal. 30 (1993), 507537.

[8] Dyn, N. and C.A. Micchelli, Piecewise polynomial spaces and geometric continuity of curves, Numer. Math. 54 (1988), 319-337.

[9] Goodman, T.N.T., Shape preserving representations, in Mathematical Methods in Computer Aided Geometric Design (T. Lyche and L.L. Schumaker eds.), Academic Press, New York, 1989, 333-357.

[10] Goodman, T.N.T., Total positivity and the shape of curves, in Total Positivity and its Applications (M. Gasca and C.A. Micchelli eds.), Kluwer Academic Publishers, 1996, 157-186.

[11] Goodman, T.N.T. , and C.A. Micchelli, On refinement equations determined by Pólya frequency sequence, SIAM J. Math. Anal. 23 (1992), 766-784.

[12] Goodman, T.N.T. , and A. Sharma, A modified Bernstein-Schoenberg operator, in Constructive Theory of Functions 87 (Bl. Sendov ed.), Bulgarian Academy Sciences, Sofia, 1988, 166-173. 
[13] Gori, L., and F. Pitolli, Multiresolution analysis based on certain compactly supported refinable functions, in Proceeding of ICAOR: International Conference on Approximation and Optimization (G. Coman, W.W. Breckner, P. Blaga eds.), Transilvania Press, 1997, 81-90.

[14] Gori, L., L. Pezza, and F. Pitolli, A class of totally positive blending B-Bases, in Proc. Curve and Surface Design: Saint Malo 1999 (P.-J. Laurent, P. Sablonnière, L.L. Schumaker eds.), Vanderbilt University Press, Nashville, TN (1999).

[15] Gori, L., and F. Pitolli, A class of totally positive refinable functions, submitted

[16] Gori, L., F. Pitolli, and L. Pezza, On the wavelet Galerkin method based on a particular class of wavelets, submitted

[17] Karlin, S., Total positivity, Stanford University Press, Stanford (1968).

[18] Micchelli, C.A., and H. Prautzsch, Refinement and subdivision for spaces of integer translates of a compactly supported function, in Num. Anal. 1987 (Griffith-Watson eds.), Longman Sc. \& Tech., Harlow (1988), 192-222.

[19] Micchelli, C.A., Y. Xu, and Y. Zhao, Wavelet Galerkin methods for second-kind integral equations, J. Comput. Appl. Math. 86 (1997), 251270 .

[20] Schoenberg, I.J., On spline functions, in Inequalities, (O. Shisha ed.), Academic Press, New York, 1967, 255-291.

[21] Schumaker, L.L., Spline functions: basic theory, Krieger Publishing Company, Malabar, Florida (1993). 